\title{
The Costs of Increasing Transparency
}

\author{
Maria Demertzis • Marco Hoeberichts
}

Published online: 1 May 2007

(C) Springer Science + Business Media, LLC 2007

\begin{abstract}
In their seminal paper, Morris and Shin (Amer Econ Rev 92(5): 1521-1534, 2002a) argued that increasing the precision of public information is not always beneficial to social welfare. Svensson (Amer Econ Rev 96: 448$451,2006)$ however has disputed this by saying that although feasible, the conditions for which this was true, were not all that likely. In that respect, therefore, increasing 'transparency' remains most of the times beneficial to social welfare. In this paper, we extend the Morris and Shin attempt by setting it up as an explicit interactive game between the Central Bank, the objectives of which we model explicitly, and the private sector. We show that in the absence of costs, both players benefit from transparency in the manner described previously in the literature, and point the differences in their gains. Following that, we then introduce the fact that increasing transparency comes at some costs and show how both players face incentives to free ride on each other as a result. The presence of costs thus alters the way in which greater transparency is attained.
\end{abstract}

Keywords Public and private signals • High order expectations • Monetary policy as an information game

JEL Classification $\mathrm{E} 31 \cdot \mathrm{E} 52 \cdot \mathrm{E} 58$

Views expressed are our own and do not necessarily reflect those of the institutions we are affiliated with.

M. Demertzis $(\varangle) \cdot$ M. Hoeberichts

Economics and Research Division, De Nederlandsche Bank,

P. O. Box 98, 1000 AB, Amsterdam, The Netherlands

e-mail: m.demertzis@dnb.nl

M. Hoeberichts

e-mail:m.m.hoeberichts@dnb.nl 


\section{Introduction}

The literature on global games (inter alia Morris and Shin 2002b; Phelps 1983; Woodford 2002) has given rise to the importance of heterogenous information in decision making. Based on Keynes' (1936) beauty context example, the argument goes, when agents need to form a view about the underlying state, they engage in trying to second-guess others' actions. This occurs because the utility they derive individually depends not only on identifying the state correctly, but also on closing the distance between their own prediction and that of others. In the context of monetary policy, this methodology is used by Amato and Shin (2003) and Amato et al. (2003) to discuss how increasing Central bank transparency (in the form of making a public signal more precise) can, under certain circumstances, prove detrimental to social welfare. Indeed this is demonstrated in the seminal paper by Morris and Shin (2002a) (MS hereafter) in which they show that this arises because in their effort to secondguess others, private agents give disproportionate more emphasis on public information that is common knowledge, than is justified by its noise. ${ }^{1}$ Svensson (2006) then argues that, true though this is, the parameterisation that is required for this to hold is very specific and actually implausible. If one was to assume plausible values he argues, then the benefits of increasing transparency are rescued and indeed dominate the range of outcomes (Blinder and Wyplosz 2005 , raise similar concerns).

In this paper we would like to take this discussion further by introducing two innovations: first, we allow explicitly for a central bank (CB) to be one, of many, contributors to what constitutes the public signal. This is because, as commonly agreed in the literature, the Central bank has an important role in coordinating private sector expectations. ${ }^{2}$ Monetary policy is now a game of two players, the central bank and the private sector, the latter being composed of an infinite number of private agents that are modelled in the MS fashion. This allows us to examine how increasing the precision of a signal (either private or public) affects the interaction between these two optimising players. Second, we then allow for the fact that increasing the precision of that signal does not come without costs. This is an important addition to the literature both because it is actually more plausible but also because it shows how the incentives of the two players may be distorted as a result, by introducing an element of free riding in their optimal actions.

The paper is organised as follows. Section 2 outlines the static game between the central bank and the private sector (PS). The central bank is a contributor

\footnotetext{
${ }^{1}$ Note that Hellwig (2004) and Angeletos and Pavan (2004) come to opposite results to the MS predictions about the benefits of increasing the precision of public information. They attribute these differences to the way the heterogeneity (of agents)-volatility (of outcomes) trade-off is modelled in these papers and the externalities it generates.

${ }^{2}$ See Blinder et al. (2001) for a general discussion on the Bank's communication role and Demertzis and Viegi (2006) for an example on how it achieves that.
} 
to the public signal (but not necessarily the sole one) and the private sector then uses what is common knowledge in combination with its own information to decide on their action. Section 3 then shows how players' welfare changes as the level of precision of the two signals increases. The underlying assumption here is that more information comes at no cost. Section 4 then lifts this assumption and introduces costs explicitly to show how the impact of greater information generates free-riding incentives. Section 5 summarises our results and concludes.

\section{Monetary policy as an information game}

The game between the two players based on the model presented in Demertzis and Viegi (2006) is summarised as follows. The central bank has a standard loss function in which it chooses the rate of inflation $x$ to minimise the distance of inflation from the target $x^{T}$ and the output gap, $y$

$$
\min _{x} L_{C B} \mid \xi=\frac{1}{2} E\left[\left(x-x^{T}\right)^{2}+y^{2}\right]
$$

subject to a standard Lucas supply function, $y=x-x^{e}+\xi$ where $\xi$ is a supply shock with zero mean and constant variance, $\sigma_{\xi}^{2}$. Note that any central bank will have an objective $x^{T}$ irrespective of whether it has communicated it to the public clearly, or even at all. Optimisation of (1) implies that

$$
\left.x\right|_{\xi}=\frac{x^{T}}{2}+\frac{x^{e}}{2}-\frac{\xi}{2}
$$

where $x$ is now the ex post inflation outcome conditional on the shock $\xi$ and $x^{e}$ is private sector expectations about the relevant rate of inflation. Representation (2) is of a structural form in the sense that expectations are not solved for Svensson (2003) argues in favour of such a representation in order to indicate that factors like judgement that contribute to the way expectations are formed but cannot always be modelled, are an important contributor to monetary policy. Note that Eq. 2 is specific to the underlying Lucas supply function assumed but demonstrates that the outcome will be a function of both the policy the Central Bank pursues as well as what the private sector anticipates. We can re-group the terms in Eq. 2 and re-write it in a general form:

$$
\left.x\right|_{\xi}=(1-r)\left(x^{T}-\xi\right)+r x^{e}
$$

where $r$ represents the strength with which expectations affect the final outcome. ${ }^{3}$ In a typical commitment game, where the central bank communicates its target $x^{T}$ and commits to it, expectations formed by all individuals

\footnotetext{
${ }^{3}$ Re-grouping the error term $\xi$ with the $\mathrm{CB}$ objective is a simplification that the model allows us to do so that we can have one term representing expectations and one the rest.
} 
collectively are equal to the CB's objectives, $x^{e}=x^{T}$ and the ex post outcome is

$$
\begin{aligned}
\left.x^{*}\right|_{\xi} & =x^{T}-(1-r) \xi \\
E\left(x^{*}\right) & =x^{T}
\end{aligned}
$$

In this paper however, we depart from the commitment set-up and allow agents to form expectations based on all the information that is available to them (therefore, inclusive of what the CB has announced). Every individual $i$ will thus be forming an expectation of inflation $x_{i}^{e}$, such that the collective outcome (for a continuum of agents) $x^{e}=\int_{0}^{1} x_{j} d j$ is the expectation that is relevant to the inflation outcome. The timing of the game assumed has the central bank deciding what its objectives are first, private agents form expectations based on information available, shocks occur next and finally the $\mathrm{CB}$ reacts to the supply shock $\xi$.

\subsection{The formation of expectations}

We thus start by arguing that while the CB may be clear itself about what its objectives are, it is not always possible to assume that private individuals form expectations that are consistent with these objectives. It becomes important then to examine the information that is available to the private sector and how they use it to form expectations. Typically, every individual forms expectations based on two information sets, namely what is publicly available and therefore common to everyone, and what is available to them privately. Furthermore, every individual is aware of the fact that the ex post outcome of inflation $x$ will be determined by Eq. 2, in other words will be affected partially by the policy the central bank pursues to attain its objectives, and partially by the average of expectations formed by the public.

However, as the individual is interested in predicting the ex post level of inflation correctly, she needs to interpret both components of Eq. 2 based on the information she has. Her objectives are captured by a standard expected dis-utility, ${ }^{4}$

$$
\min _{x_{i}^{e}} u_{i}\left(\mathbf{x}^{e}, x^{T}\right) \equiv \frac{1}{2} E_{i}\left(x_{i}^{e}-x\right)^{2}
$$

Note that subscript $i$ in the expectations operator, indicates that the individual will be seeking to minimise her expected dis-utility, given her own perceptions.

\footnotetext{
${ }^{4} \mathrm{We}$ assume that the individual consumer sets a price variable (individual wage) and supply elastically to the amount of labour demanded. This is just a narrative trick: the argument would work equally well in a set up as in Lucas' island model in which individuals set the price of a good in an imperfect knowledge set-up, (see Canzoneri 1985).
} 
$x_{i}^{e}$ is individual $i$ 's expectation of what inflation will be and $x$ is again the ex post inflation outcome from Eq. 3. We use $\mathbf{x}^{e}$ to refer to the expectations profile over all agents. The objective of each individual $i$ is thus to form expectations $x_{i}^{e}$, as accurately as possible, which she will then use, for example, in wage negotiations. The individual decides her action $x_{i}^{e}$ based on the first-order condition of Eq. 6. This is:

$$
\arg \min _{x_{i}^{e}} u_{i}\left(\mathbf{x}^{e}, x^{T}\right)=E_{i}(x)
$$

and from Eq. 3,

$$
\begin{aligned}
x_{i}^{e} & =E_{i}(x) \\
x_{i}^{e} & =E_{i}\left[(1-r)\left(x^{T}-\xi\right)+r x^{e}\right] \\
x_{i}^{e} & =(1-r) E_{i}\left(x^{T}-\xi\right)+r E_{i}\left(x^{e}\right)
\end{aligned}
$$

The optimal action for individual $i$ is thus a function of three things: the objectives of the central bank and hence the policy it will pursue, the shock that will occur, and finally the average expectation formed by all individuals. Moreover, in forming expectations $x_{i}^{e}$, individual $i$ needs to evaluate these three things, captured here by the expectations operator, subscript $i .^{5}$ It follows that if $x_{i}^{e}=x_{j}^{e} \forall j$, then $x_{i}^{e}=x^{e}$ and individuals' expectations are matched. However, although desirable, coordination between agents at any level of inflation is not sufficient; the optimal outcome occurs when agents coordinate at the objective pursued by the central bank. Coordination at any other expectation rate still leaves agents away from the level of inflation that the $\mathrm{CB}$ aims to achieve. Following Morris and Shin (2002a), we argue that information used by the agents is available in the form of a public signal common to all, and a private signal which is specific to each agent in the economy. These take the following form:

$$
\begin{gathered}
\text { Public signal: } p=\left(x^{T}-\xi\right)+\eta \\
\text { Private signal: } z_{i}=\left(x^{T}-\xi\right)+\varepsilon_{i}
\end{gathered}
$$

Both $\eta$ and $\varepsilon_{i}$ are normally distributed with zero mean and variance $\sigma_{\eta}^{2}$ and $\sigma_{\varepsilon}^{2}$ respectively. Furthermore, the two error terms are independent of $x$ and of each other, such that $E\left(\varepsilon_{i} \varepsilon_{j}\right)=0$ for $i \neq j$. Contrary to MS then, the clarity

\footnotetext{
${ }^{5}$ Equation 7 is not dissimilar to Eq. 2 of Morris and Shin (2002a) in which the individual forms a view about the state $\theta$ and the average action, $\bar{a}$. The strength with which she pursues that is given by the "beauty term" parameter $r$ provided by the model. An important difference to the Morris and Shin (2002a) approach however, is that inflation outcome $x$ is now endogenous, in the sense of being affected by the average action, whereas in the MS approach $\theta$ is a state variable independent of $\bar{a}$.
} 
of public information is not under the full control of the $\mathrm{CB}$ but is affected by a combination of the CB's information strategy, general market information available and noise. Based on these two types of signals, MS show that action for agent $i$, applying Eqs. 8 and 9 on Eq. 7 then gives,

$$
\begin{aligned}
x_{i}^{e} & =\frac{\alpha p+\beta(1-r) z_{i}}{\alpha+\beta(1-r)} \\
& =x^{T}-\xi+\frac{\alpha \eta+\beta(1-r) \varepsilon_{i}}{\alpha+\beta(1-r)}
\end{aligned}
$$

where $\alpha=\frac{1}{\sigma_{\eta}^{2}}$ and $\beta=\frac{1}{\sigma_{\varepsilon}^{2}}$ represent the precision for the two information sets, respectively. We call this the MS action. It follows that expectations across all agents are then equal, to

$$
x^{e}=\int_{0}^{1} x_{j} d j=x^{T}-\xi+\frac{\alpha \eta}{\alpha+\beta(1-r)}
$$

Equation 11 shows that the average expectation across all agents will be distorted by the (lack of) precision of the two signals as well as the value for the 'beauty term' $r$. For simplification purposes, we drop $\xi$ as it does not change the qualitative nature of our results. The timing of the game then implies that the CB provides a public signal based on which the PS then forms expectations. In turn, this implies that expectations are predetermined at the time the central bank is asked to take action. In other words, the central bank needs to implement the policy rule it had announced in its signal, subject to Eq. 11,

$$
\begin{aligned}
L_{C B} & =E\left[\left(x-x^{T}\right)^{2}+\left(x-x^{e}\right)^{2}\right] \\
\text { st. } \quad x^{e} & =x^{T}+\frac{\alpha \eta}{\alpha+\beta(1-r)}
\end{aligned}
$$

Note that average expectations across all possible public signals are

$$
E\left(x^{e}\right)=\int_{-\infty}^{+\infty}\left(x^{T}+\frac{\alpha \eta}{\alpha+\beta(1-r)}\right) d \eta=x^{T}
$$

As $E\left(x^{*}\right)=E\left(x^{e}\right)=x^{T}$ then Eq. 4 is indeed the optimal reaction consistent with Rational Expectations. We calculate next the losses incurred ex post by the two players. CB losses are found by substituting Eqs. 4 and 11 into Eq. 1: ${ }^{6}$

$$
\begin{aligned}
L_{C B} & =E\left[\left(x^{*}-x^{T}\right)^{2}+\left(x^{*}-x^{e}\right)^{2}\right] \\
& =\frac{\alpha}{[\alpha+\beta(1-r)]^{2}}
\end{aligned}
$$

\footnotetext{
${ }^{6}$ Note that since $E\left(x^{*}\right)=E\left(x^{e}\right)=x^{T}$ the Central Bank is unable ex ante to distinguish between expectations in Eq. 11 and the commitment case, Eq. 5, which leads to Eq. 4. When calculating the losses incurred, one needs to consider the ex post expectations formation.
} 
Similarly, from Morris and Shin (2002a) we know that losses for the private sector collectively, ${ }^{7}$ are:

$$
\begin{aligned}
L_{P S} & =\int_{0}^{1}\left(x_{i}^{e}-E\left(x^{*}\right)\right)^{2} d i \\
& =\frac{\alpha+\beta(1-r)^{2}}{[\alpha+\beta(1-r)]^{2}}
\end{aligned}
$$

It is straightforward to show that the slope of losses for both the central bank as well as society is not unequivocally signed in terms of changes in the precision of the public signal but depends on the parameterisation of $r$. We examine next how the incentives of the central bank and the private sector may be affected, if transparency (i.e. the precision of the two signals) was subject to choice.

\section{Information and welfare}

\subsection{The role of strategic motive $r$}

We first examine how the losses for the two players are affected by term $r$, the private sector's strategic motive to be as close to average expectations as possible.

Proposition 1 As private agents' strategic motive increases, $(d r>0)$, both social as well as $C B$ welfare deteriorate.

Proof We can demonstrate this by looking at the way losses vary with $r$.

$$
\begin{aligned}
L_{C B} & =\frac{\alpha}{[\alpha+\beta(1-r)]^{2}} \\
\frac{\partial L_{C B}}{\partial r} & =\frac{2 \alpha \beta}{[\alpha+\beta(1-r)]^{3}}>0
\end{aligned}
$$

\footnotetext{
${ }^{7}$ Note that the extent to which aggregate losses for the private sector differ from that of the Central Bank (who is by definition optimising for the whole of the society) reflects the inability of the individual to internalise the aggregate welfare in her optimisation process. Her optimal choice therefore, and by consequence that of everyone else individuallly, do not necessarily amount to society's optimal.
} 
Similarly for the private sector,

$$
\begin{aligned}
L_{P S} & =\frac{\alpha+\beta(1-r)^{2}}{[\alpha+\beta(1-r)]^{2}} \\
\frac{\partial L_{P S}}{\partial r} & =\frac{2 \alpha \beta r}{[\alpha+\beta(1-r)]^{3}}>0
\end{aligned}
$$

The intuition behind this result is that the externality of the 'beauty contest' term moves the action away from the otherwise optimum. A positive value of $r$ induces private sector agents to reduce the weight attached to their private information by $(1-r) \beta$. So, as $r$ increases, ${ }^{8}$ the weight that the private sector puts on the public signal is greater than what is justified by the relative precision of the two signals. Similarly, for the central bank, the increase in the private sector's strategic motive increases the distance between its inflation objective and the expectations that the private sector forms. Two further comparisons are worth mentioning. First, irrespective of parameterisation, there will always exist a range of values for $\alpha$ for which the central bank incurs higher losses by increasing the precision of the public signal further (i.e. $\frac{\partial L_{C B}}{\partial \alpha}>0$ for $\left.\alpha \in(0, \tilde{\alpha})\right)$. For the private sector this is only true for a very specific choice of parameter values, outwith which, increasing $\alpha$ is always beneficial to its welfare. Second, comparing the two deteriorations in losses, the $\mathrm{CB}$ in fact stands the most to lose because while it incurs the costs of this increased externality, it does not face any of the benefits associated with this extra term (incorporated in the individual's utility function). It is straightforward to see that by comparing Eq. 17 to Eq. 16. We turn next to how increasing the public and private signal precision affects the losses of the two players. Implicit in our approach is the idea that the exact level of precision is subject to choice for the two players accordingly.

\subsection{Public signal}

We first demonstrate how increases in the precision of the public signal (identified often with increases in transparency) affect the two players' losses in the game.

$$
\begin{aligned}
\frac{\partial L_{C B}}{\partial \alpha} & =\frac{-\alpha+\beta(1-r)}{[\alpha+\beta(1-r)]^{3}} \\
\frac{\partial L_{P S}}{\partial \alpha} & =-\frac{\alpha+\beta(r-1)(2 r-1)}{[\alpha+\beta(1-r)]^{3}}
\end{aligned}
$$

\footnotetext{
${ }^{8}$ Quoting Krugman (2001), Morris and Shin (2002a) argue that the value of $r$ may have increased over the recent years.
} 
As argued by MS, increasing the level of precision of the public signal will not always be beneficial to the private sector (or indeed the central bank as we show here), as expressions (18) and (19) are not unequivocally signed. We summarise next the conditions required for the derivatives to sign one way. Starting with the central bank, for transparency to be detrimental to its losses, (i.e., $\frac{\partial L_{C B}}{\partial \alpha}>0$ ), the necessary condition is that

$$
\begin{aligned}
\beta(1-r) & >\alpha \\
(1-r) & >\frac{\alpha}{\beta}
\end{aligned}
$$

The $\mathrm{CB}$ will always have a hump shaped function such that the optimal level is at $\alpha=0$; but once this is violated (for example, because the central bank is only one of many contributors to public information and not the exclusive one), then it is always optimal to try to increase $\alpha$ as much as possible such that losses return to zero. As, we will show in the next section, this result does not hold if increasing the public signal is not without cost. Turning next to the private sector, the necessary condition for social losses to be increasing in transparency, i.e. $\frac{d L_{P S}}{d \alpha}>0$, (as discussed by MS and Svensson 2006) is that

$$
\begin{aligned}
f(r) & >\frac{\alpha}{\beta} \\
\text { where } \quad f(r) & \equiv(1-r)(2 r-1)
\end{aligned}
$$

For Eq. 21 to be true, a necessary condition is that parameter

$$
r \in\left(\frac{1}{2}, 1\right)
$$

If $r \in\left[0, \frac{1}{2}\right]$ or $r=1$ then $(1-r)(2 r-1) \leq 0$ and Eq. 21 is violated. This implies that for transparency to be detrimental to welfare, agents must put a sufficiently high (although not exclusive) weight on the 'beauty contest' term. However, beyond that Svensson (2006) also mentions that $f(r)$ has a maximum equal to $\frac{1}{8}$ when $r=\frac{3}{4}$. This implies that even if Eq. 22 holds, condition (21) maybe violated if

$$
\frac{\alpha}{\beta} \geq \frac{1}{8}
$$

In other words if the public signal precision is greater than one eighth of the private signal precision, social welfare benefits from increases in transparency. Moreover, if $r$ is on the other side of $\frac{3}{4}$ but satisfying condition (22), then the likelihood of transparency being beneficial is even greater.

Naturally, the same holds for the private sector, even if for some parameterisations it has to put up with increasing losses before it starts to benefit from an increasing $\alpha$ again. 
Fig. 1 Losses against public signal precision $(r=0.7)$

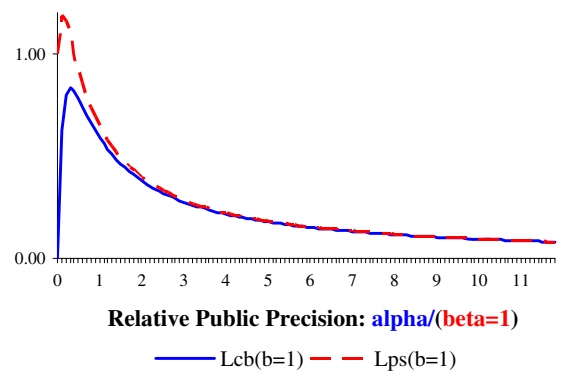

Figure 1 demonstrates this point for $r=0.7$, for which both the CB as well as the PS have to put up with a deterioration in their welfare for increases within a given range of public signal precision (see Appendix A for alternative parameterisation of $r$ ). Note that for $\alpha=0$, the central bank is at its optimal point whereas the PS is at a positive level of losses. As mentioned earlier, this maybe the optimal level for the central bank but it is a very unlikely outcome (Svensson 2006).

Furthermore, it is also true that increases in transparency, do not affect the two players in the same way. It follows that:

Proposition 2 For any increase in the precision of the public signal, the private sector benefits more (or loses less) than the central bank.

Proof It is straight forward to prove this by comparing Eq. 18 to Eq. 19 by taking the difference between the two

$$
\begin{aligned}
\frac{\partial L_{C B}}{\partial \alpha}-\frac{\partial L_{P S}}{\partial \alpha} & =\frac{-\alpha+\beta(1-r)}{[\alpha+\beta(1-r)]^{3}}-\frac{-\alpha+\beta(1-r)(2 r-1)}{[\alpha+\beta(1-r)]^{3}} \\
& =\frac{2 \beta(1-r)^{2}}{[\alpha+\beta(1-r)]^{3}}>0
\end{aligned}
$$

Figure 2 also demonstrates this for a given parameterisation of $r(=0.6)$.

Fig. 2 Marginal losses against public signal precision $(\mathrm{r}=0.6)$

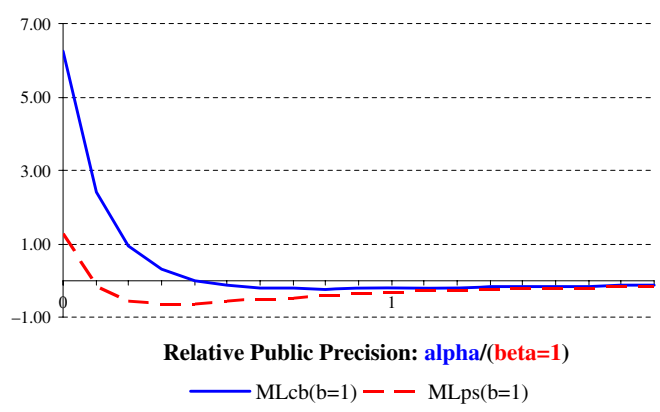


Coupled with the fact that, for plausible parameterisations, increases in transparency are always beneficial to the private sector, this proposition implies that private agents are justified to lobby for increases in $\alpha$ from the central bank or even for any other source that contributes to public information.

\subsection{Private signal}

The effects of increases in the precision of private information are different in that both parties benefit from the increase in its precision demonstrated by

$$
\begin{aligned}
\frac{\partial L_{C B}}{\partial \beta} & =\frac{2 \alpha(r-1)}{[\alpha+\beta(1-r)]^{3}} \leq 0 \\
\frac{\partial L_{P S}}{\partial \beta} & =\frac{(r-1)\left[\beta(1-r)^{2}+\alpha(1+r)\right]}{[\alpha+\beta(1-r)]^{3}} \leq 0
\end{aligned}
$$

This can also be seen in Fig. 3 for a given parameterisation of $r(=0.7)$ but holds also in general.

However, the difference between the two is not unequivocally signed and thus there are instances for which one of the two players benefits the most.

$$
\begin{aligned}
\frac{\partial L_{C B}}{\partial \beta}-\frac{\partial L_{P S}}{\partial \beta} & =\frac{2 \alpha(r-1)}{[\alpha+\beta(1-r)]^{3}}-\frac{(r-1)\left[\beta(1-r)^{2}+\alpha(1+r)\right]}{[\alpha+\beta(1-r)]^{3}} \\
& =-\frac{[\alpha+\beta(r-1)](r-1)^{2}}{[\alpha+\beta(1-r)]^{3}}
\end{aligned}
$$

The important point of this section however, is that for plausible (and even conservative) parameter values both in terms of $r$ as well as ratios of public to private signal precision, increasing the levels of precision of either of the players's signal is beneficial to both. We examine next how (and whether) this may be distorted if one, or possibly both had to incur costs in order to increase the precision of their own signal.

Fig. 3 Losses against private signal precision $(\mathrm{r}=0.7)$

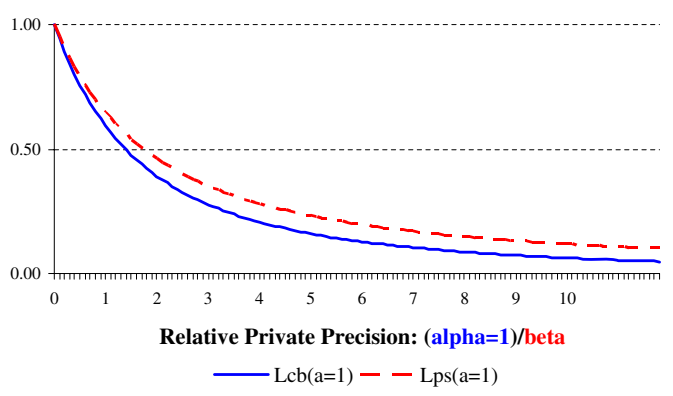

国 Springer 


\section{Introducing costs}

The analysis so far assumes that there are no costs associated with acquiring information that contributes to the increase of the precision of the signal (either public or private). We consider this rather implausible as we believe that acquiring greater information is always costly. This argument is of course relevant when seen from the private sector's point of view but is it also relevant for a Central Bank. It is argued that as the Central Bank is intended to pursue the public's interest then, for any given resources, it should strive to acquire as much information as possible so that it takes the most informed decision. Naturally for any given amount of information it has, the Central Bank always retains a certain degree of discretion in terms of what level of transparency it operates at. The point that we wish to raise next however, is that if information is costly to acquire, then the Central Bank (and the private sector) is quicker to stop looking to increase it for itself. Moreover, this gives rise to a "free-ride" effect from both sides, in the sense that both players prefer the other to invest in increasing the information set available. We show this next.

We assume that costs are positive and unbounded and adopt a general BoxCox transformation.

$$
\begin{aligned}
C_{C B} & =c_{C B} \alpha^{-\lambda} \\
C_{P S} & =c_{P S} \beta^{-\lambda}
\end{aligned}
$$

where $c_{C B}>0$, and $c_{P S}>0, \lambda$ is negative. For $\lambda=-1$ the marginal cost is constant (linear), and $\lambda=-2$ (quadratic) is the functional form applied by Svensson (2006), who assumes increasing marginal costs. The rationale behind Svensson's choice is that information is increasingly costly to acquire ('...producing better forecasts requires more and better -qualified staff'). Each of the two agents now faces the sum of welfare losses and the costs of increasing the level of precision of the signal.

$$
T=L+C
$$

In what follows we assume that costs are linear, $(\lambda=-1)$, for analytical tractability, but we can generalise our results for all values of $\lambda<0$ (see Appendix B).

\subsection{Linear costs $(\lambda=-1)$}

We assume first, that it is only the $\mathrm{CB}$ that incurs costs in increasing the precision of the public signal. The private sector receives information some of which is common to everyone and some specific, but does not incur any costs. Furthermore, we argue that the $\mathrm{CB}$ is one, of many contributors to public information and therefore, it is reasonable to assume that $\alpha>0$. Faced therefore, with a certain positive level of precision, we examine next how the costs of increasing this information affects the CB' $s$ incentives to increase transparency. 
Fig. 4 Losses and total (linear costs against public signal $)(r=0.5)$

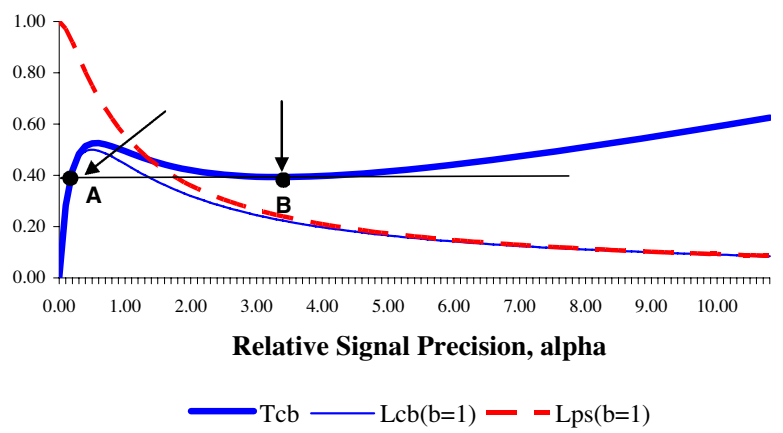

Figure 4 shows that the existence of costs, clearly prevents the $\mathrm{CB}$ from increasing the level of precision of public information indefinitely. Furthermore, it is also clear, that if the level of precision of public information is to the left of point $A,{ }^{9}$ then the $\mathrm{CB}$ will not try to increase it, as by doing so, it increases its losses. However, if public information is to the right of point $A$ then the CB does have an incentive to increase the level of precision up to point $B$. Beyond that however, the costs of going further outweigh the benefits of doing so. It is worth noting that as the degree of non-linearity increases $(\lambda \downarrow)$ then both the total costs as well as marginal costs increase (i.e. $C_{\lambda}^{\prime}<0$, and $C_{\lambda}^{\prime \prime}>0$ ). This implies that the cut-off point $B$ beyond which the central bank would not wish to increase the level of precision any longer is displaced leftwards (reduced, see Appendix B). Beneficial though this is to society's welfare, the CB is faced with a local minimum which distorts its incentives to increase its contribution to public information. Naturally, if public information was to increase through some other source, then the $\mathrm{CB}$ would benefit in the same manner as described in the previous section.

We assume next that both the central bank as well as the private sector incur (linear) costs when wishing to improve the quality of their signal. Both players are then faced with having to minimise the following:

$$
\begin{aligned}
T_{C B} & =L_{C B}+C_{C B} \\
T_{P S} & =L_{P S}+C_{P S}
\end{aligned}
$$

An optimising central bank is now faced with the following first-order condition:

$$
\frac{\partial T_{C B}}{\partial \alpha}=\frac{-\alpha+\beta(1-r)}{[\alpha+\beta(1-r)]^{3}}+c_{C B}=0
$$

\footnotetext{
${ }^{9}$ Point $B$ is the local minimum that satisfies the first and second-order conditions for the optimal level of precision of public information, which we label $\alpha^{*}$. Since $T_{C B}(0)=0$, point $A$ is found by solving $T_{C B}(\alpha)=T_{C B}\left(\alpha^{*}\right)$.
} 
Ensuring that optimisation leads to minimisation of the function, requires that the second-order condition is met, i.e.

$$
\frac{\partial^{2} T_{C B}}{\partial \alpha^{2}}=\frac{2(\alpha-2 \beta(1-r))}{[\alpha+\beta(1-r)]^{4}}>0
$$

For Eq. 29 to be true, the sufficient condition is that $\alpha>2 \beta(1-r)$ holds. However, in equilibrium, as both conditions need to be satisfied, the following restriction needs also to apply.

$$
c_{C B}>\frac{\beta(1-r)}{[\alpha+\beta(1-r)]^{3}}
$$

The first-order condition is a third-order polynomial and solving it explicitly for an optimal level of precision of central bank information $\alpha$ does not provide much insight. However, we can apply the implicit function theorem to characterise the strategic substitutability between the two signals in equilibrium.

Proposition 3 In equilibrium, the optimal value of public signal precision, as affected by the central bank, is decreasing in the precision of the private signal.

Proof Let $F:=\frac{\partial T_{C B}}{\partial \alpha}$; then the implicit function theorem implies that

$$
\frac{\partial \alpha^{*}}{\partial \beta}=-\frac{F_{\beta}}{F_{\alpha}}=\frac{(1-r)(2 \alpha-\beta(1-r))}{2 \beta(1-r)-\alpha}
$$

The restriction on the second-order condition $(\alpha>2 \beta(1-r))$, implies that it is straight forward to show that $\frac{\partial \alpha^{*}}{\partial \beta}<0$. In other words, if the private sector reduces the level of precision of its own signal, then in order to maximise its utility, the $\mathrm{CB}$ is forced to increase its contribution to the public signal, in equilibrium.

Turning to the private sector, the FOC is now

$$
\frac{\partial T_{P S}}{\partial \beta}=\frac{-(1-r)\left[\beta(1-r)^{2}+\alpha(1+r)\right]}{[\alpha+\beta(1-r)]^{3}}+c_{P S}=0
$$

It is straight forward to demonstrate that the second-order condition is always met for the private sector.

$$
\frac{\partial^{2} T_{P S}}{\partial \beta^{2}}=\frac{2(1-r)^{2}(\alpha+\beta(1-r)+2 \alpha r)}{[\alpha+\beta(1-r)]^{4}}>0
$$


Proposition 4 In equilibrium, the optimal value of private signal precision, as dictated by the representative private agent, is decreasing in the precision of the public signal.

Proof Let $G:=\frac{\partial T_{P S}}{\partial \beta}$; then the implicit function theorem implies that

$$
\frac{\partial \beta^{*}}{\partial \alpha}=-\frac{G_{\alpha}}{G_{\beta}}=-\frac{\beta+\alpha(1+r)+\beta r(2 r-3)}{(1-r)\left(\alpha+\beta(1-r)^{2}+2 \alpha r\right)}
$$

The restriction for the central bank's second-order condition $(\alpha>2 \beta$ $(1-r))$ helps show that $\frac{\partial \beta^{*}}{\partial \alpha}<0$. Similarly to the central bank, if the precision of public information increases, it is optimal for the private sector to reduce the precision of its own information.

In summary, the result above shows that, provided the first-order conditions for both the central bank and the private sector and the second-order condition for the central bank are satisfied, public and private information are strategic substitutes in line with Morris and Shin's contribution. However, as pointed out by Svensson with reference to this set-up, it is very unlikely that providing public information is detrimental to social welfare in the absence of costs. When introducing costs on the other hand, strategic substitutability is a very likely outcome.

\section{Conclusions}

We have presented a model where the $\mathrm{CB}$, as a provider of public information and private sector agents, as providers of private information, interact strategically. First, we replicate the result found by MS that, in the presence of the socalled "beauty-contest externality" that arises when private sector agents try to second-guess other private sector agents' expectations, more precise public information may be detrimental to welfare. As a next step, we attach costs to the provision of more precise public and private information. When these costs are taken into account, we find an optimal level for the precision of private information and a local optimum for the precision of public information. The global optimum is reached for zero precision of public information, confirming the result by MS. However, as pointed out by Svensson, since the CB is not the only provider of public information, it is implausible that the global optimum can be reached, and very well possible that the local optimum is the best strategy for the CB. We derive the exact conditions under which this is the case.

Our second contribution is the strategic substitutability between the precision of public and private information that arises once we take the costs of providing information into account. We show that, in equilibrium, a marginal increase in the precision of the private signal induces the central bank to reduce the precision of the public signal. The reverse also holds: A marginal increase 
in the precision of the public signal induces the private sector to reduce the precision of its information.

Acknowledgements Without wishing to implicate, we would like to thank participants at the 10th ICMAIF conference and in particular our discussant Friederike Köhler, Peter van Els and Lex Hoogduin and seminar participants at the University of Amsterdam for comments and suggestions. The usual disclaimer applies.

\section{Appendices}

\section{A The value of $r$}

We see that the $\mathrm{CB}$ faces the hump-shape losses for all values of $r$. By contrast, as mentioned in the main text, the private sector benefits from greater transparency for a big number of values of $r$. Given the choice of values for the public and private information signal, the private sector faces a hump-shape loss function only for $r>0.7$ (see Fig. 5).

Fig. 5 a CB losses against public signal precision (varying $r$ ). b PS losses against public signal precision (varying $\mathrm{r}$ ) a

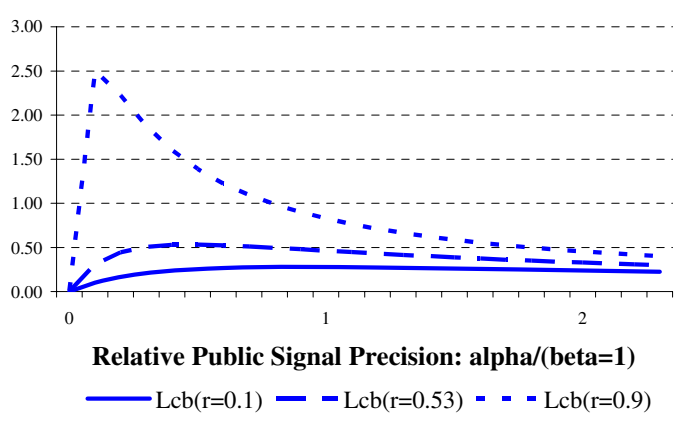

b

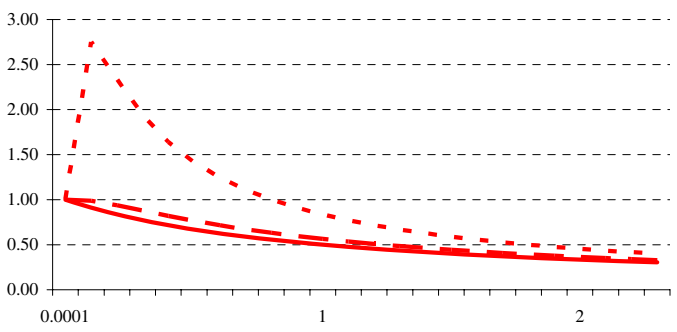

Relative Public Signal Precision: alpha/(beta=1) 


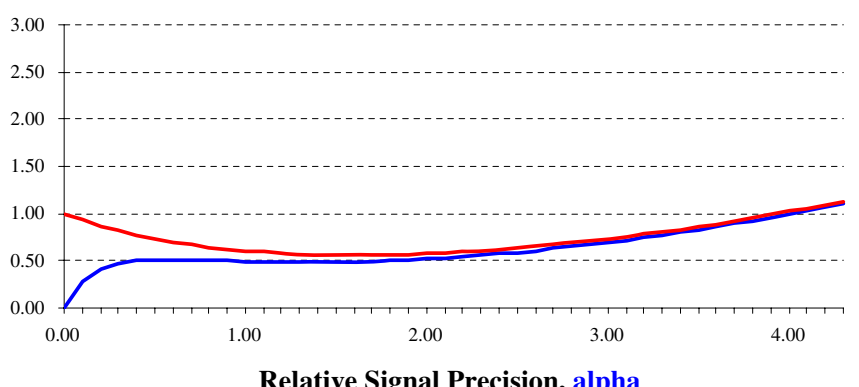

Relative Signal Precision, alpha

$-\mathrm{Tcb}^{\wedge}{ }^{2} \quad-\mathrm{Tps}^{\wedge} 2$

Fig. 6 Quadratic costs: losses for CB and PS against public signal

\section{B Non-linear costs}

We have assumed linear costs in the main text. Naturally, as the degree of nonlinearity increases, then increasing the level of signal precision is not always beneficial for neither the Central Bank nor the private sector (see Fig. 6).

An optimising central bank is now faced with the following firstorder condition:

$$
\frac{\partial T_{C B}}{\partial \alpha}=\frac{-\alpha+\beta(1-r)}{[\alpha+\beta(1-r)]^{3}}-\lambda c_{C B} \alpha^{-\lambda-1}=0
$$

Ensuring that optimisation leads to minimisation of the function requires that the second-order condition is met, i.e.

$$
\frac{\partial^{2} T_{C B}}{\partial \alpha^{2}}=\frac{2(\alpha-2 \beta(1-r))}{[\alpha+\beta(1-r)]^{4}}+\lambda(\lambda+1) c_{C B} \alpha^{-\lambda-2}>0
$$

As in the linear case, the second-order condition for a minimum is always met if $\alpha>2 \beta(1-r)$. Combined with the first-order condition (and since $\lambda<$ $0)$, a minimum will always exist if:

$$
c_{C B}>\frac{\beta(1-r)}{-\lambda \alpha^{-\lambda-1}[\alpha+\beta(1-r)]^{3}}
$$

Fig. 7 Total losses linear and quadratic, against public signal $(r=0.5)$

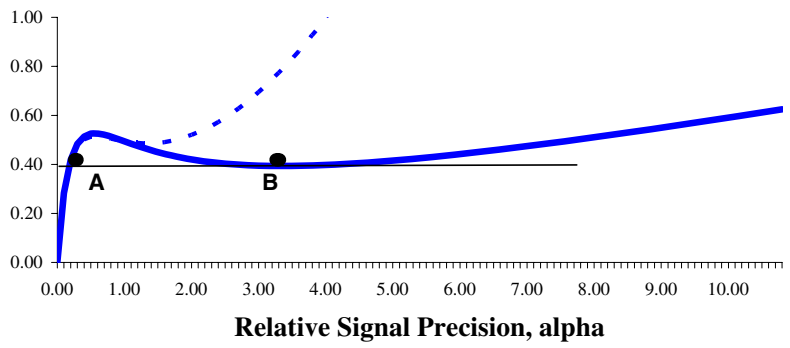


And the range of values between A and B in Fig. 7, where the Central Bank will always want to increase the level of public signal precision is reduced.

\section{References}

Amato JD, Morris S, Shin HS (2003) Communication and monetary policy. Oxf Rev Econ Policy 18(4): 495-503

Amato JD, Shin HS (2003) Public and private information in monetary policy models. BIS Working Papers, no. 138, September

Angeletos G-M, Pavan A (2004) Transparency of information and coordination in economies with investment complementarities. Amer Econ Rev 94(2): 91-98

Blinder A, Goodhart C, Hildebrand P, Lipton D, Wyplosz C (2001) How do Central Banks talk? Geneva Report on the World Economy, no. 3, London, Centre for Economic Policy, Research/International Centre for Monetary and Banking Studies

Blinder A, Wyplosz C (2005) Central bank talk: committee structure and communication policy. Working paper, prepared for the ASSA meeting, Philadelphia, January 7-9

Canzoneri M (1985) Monetary policy games and the role of private information. Amer Econ Rev 75(5): 1056-70

Demertzis M, Viegi N (2006) Inflation targets as focal points. DNB working Paper, no. 17, Version (May 2006) http://www1.fee.uva.nl/toe/content/people/demertzis.shtm.

Hellwig C (2004) Heterogenous information and the benefits of transparency. UCLA, Mimeo, December

Keynes JM (1936) The general theory of employment interest and money. Macmillan, London

Krugman P (2001) Out of the loop. New York Times, March 4, Sec. 4, p. 15

Morris S, Shin HS (2002a) Social value of public information. Amer Econ Rev 92(5): 1521-1534 December

Morris S, Shin HS (2002b) Global games: theory and applications. In: Dewatripont M, Hansen L, Turnovsky S (eds) Advances in economics and econometrics, the eighth world congress. Cambridge University Press, Cambridge, UK

Phelps ES (1983) The trouble with rational expectations and the problem of inflation stabilisation. In: Frydman R, Phelps ES (eds) Individual forecasting and aggregate outcomes. Cambridge University Press, New York, pp 31-40

Svensson L (2003) What is wrong with Taylor rules? Using judgement in monetary policy through targeting rules. J Econ Lit vol XLI: 426-477, June

Svensson L (2006) Social value of public information: Morris and Shin (2002) Is actually pro transparency, not con. Amer Econ Rev 96: 448-451

Woodford M (2002) Imperfect common knowledge and the effects of monetary policy. In: Aghion P, Frydman R, Stiglitz J, Woodford M (eds) Knowledge, information, and expectations in modern macroeconomics: In honor of Edmund S. Phelps, Princeton Univ. Press, Princeton 